\title{
Effect of some natural oils on storage and quality of Balady mandarin fruits
}

\author{
El-Salhy, A.M., A.A. Badawy, R.A. Ibrahim and M.G. Mohamed* \\ Department of Pomology, Faculty of Agriculture, Assiut University, Assiut 71526, Egypt;
}

\begin{abstract}
This study was conducted during two successive seasons 2017 and 2018 to study the effect of some oils immersing on the storability and quality of Balady mandarin (Citrus reticulate $L$.) fruits during the storage period. The post-harvest treatments were immersed with camphor oil, linen oil and imazalil either singly or in combination. The fruits were stored at cold condition $8 \pm 1^{\circ} \mathrm{C}$ with $85-90 \% \mathrm{RH}$. Samples of each treatment were randomly withdrawn monthly for 4 months. The results showed that fruit weight loss $\%$, fruit decay $\%$, total soluble solids $\%$, sugar contents and TSS/acid ratio were significantly increased with prolonging the storage period. On other hand, the advanced storage period induced a gradual decrease of fruit juice percentage, fruits firmness, acidity $\%$ and vitamin C. All treatments caused a decrease in fruit weight and fruit decay percentage, fruits firmness, acidity $\%$ and vitamin $\mathrm{C}$. Using oils resulted in the least fruit weight loss, whereas, imazalil treatment had the least fruit decay percentage. Also, all treatments improved the fruit quality during the storage period compared control. It could be concluded that oils plus imazalil coating maintained fruit freshness without negative effects of fruit quality parameters and seems to be the proper and ideal treatment to prolong cold storage of Balady mandarin fruits without great reduction in fruit quality.
\end{abstract}

Keywords: Camphor oil, Linin oil, Imazalil, Balady mandarin, Storage.

\section{Introduction}

Citrus is one of the most important crops in the world. It ranks the third position between fruit crops and only proceeds with grapes and apple. Citrus is the leading fruit crops occupying first rank among fruit crops in Egypt. Balady mandarin is considered one of the most important citrus species that Egyptian consumers prefer because of its easily peeling than other citrus species. No doubt that process of handling and storage

*Corresponding author: Mostafa Galal

Email: mgm.most@gmail.com

Tel.: +201069057256

Received: February 23, 2020;

Accepted: March 31, 2020;

Published: April 1, 2020. for local or export market is an important interest to maintain fruit quality. Postharvest diseases deteriorate $10-30 \%$ of the total yield of the fruit crops. In developing countries, improper post-harvest handling destroys more than $30 \%$ of the fresh produced horticulture crops (Kader, 2002 and Mansour et al., 2014). Citrus fruits suffer from relatively high losses during the harvesting and handling chain, i.e. unideal post-harvest treatments (storage conditions and coating material) in addition to pathological infections which substantially contribute in this crop losses and deterioration of quality after harvest (Eman and Magda, 2006 and Barakat et al., 2015). 
Traditionally, plant diseases control is achieved mainly through the use of fungicides, post-harvest due to decay may increase up to $50 \%$ without fungicide treatment, although decay can be reduced to $5-10 \%$ with post-harvest fungicides. Synthetic fungicides such as Imazalil thiabendazole and sodium ortho-phenyl phonate have been used traditionally to control post-harvest diseases is an effective method for preserving quality and extending shelf life (Poppe et al., 2003 and Mehrdad and Sajad, 2012).

The use of synthetic chemicals to control post-harvest decay has been restricted to few fruits and vegetables, due to their high and acute residual toxicity, environmental pollution, effects on food and other sideeffects on humans (Tripathi and Duby, 2004). Thus, the development and use alternative post-harvest control options involving natural plant extracts have become important since it is perceived as being environmentally safer and more acceptable to the general public (Janisiewicz and Korsten, 2002; Bajpai et al., 2008 and Gouda-Fatma-El-Zahraa and Badawy, 2016).

Consumers are demanding less chemical residue on produce, and many fungi are developing resistance to commonly used fungicides (Ragsdale and Sisler, 1994; Bull et al., 1997). Recently, researchers have shown an interest in the application of nontoxic alternatives or supplements to synthetic fungicides. Plant extracts, including essential oils, have been investigated as alternative measures against pathological breakdown (Klieber et al., 2002; Ahmed et al., 2007; Badawy-Ibtesam et al., 2011; Zaghloul et al., 2011 and Barakat et al., 2015

Coating fruit has been associated with protect perishables from deterioration by reducing transpiration and respiration, improving textural quality, retaing colour and volatile flavour compounds and reducing microbial growth (MahmoudThanaa et al.2017, Bakhtiarizade and souril, 2019, Taslima et al. 2020 and Ennab et al. 2020)

Thus, the main goal of this study was to investigate the effect of -dipping balady mandarin fruits with camphor oil, linen oil either as single treatment or combined with Imazalil as a chemical fungicide on postharvest fruits quality attributes during cold storage for 4 monthes.

\section{Materials and Methods}

The present work was carried out on Balady mandarin during 2017 and 2018 seasons. The fruits were obtained from the experimental orchard of Faculty of Agriculture, Assiut University, Egypt. The fruits were harvested at maturity stage and directly transferred to the laboratory. Defective fruits including wounded and other physiological and pathogenic disorders were excluded. The rest of uniform fruits were washed with distilled water, dried and then immersed in the following treatments:

1 - $10 \%$ camphor oil for 5 minutes.

2- $10 \%$ linen oil for 5 minutes.

3- 1000 ppm Imazalil (IMZ) for 2.5 minutes.

4- $10 \%$ camphor oil + 1000 ppm IMZ for 2.5 minutes.

5- $10 \%$ linen oil +1000 ppm IMZ for 2.5 minutes.

6- distilled water for 5 minutes (control). 
After immersing, fruits of each treatment were air dried and packed in one layer inside well aerated carton boxes $(30 \times 40 \times 12 \mathrm{~cm})$ and stored at $8 \pm 1{ }^{\circ} \mathrm{C}$ and85$90 \%$ RH. Each treatment was comprised three boxes (20 fruits/box). Fruits of each treatment were evaluated each month (4 times interval) throughout the storage period (4 months). Changes of some physical and chemical fruits characteristics were determined

\section{Physical characteristics:}

1- Weight Loss \%: It was calculated as percentage from the initial weight.

2- Fruit Decay \%: It was determined by counting the number of decay fruits (pathological or physiological disorders) throughout the four months and expressed as a percentage of the initial number of fruits per each sample (replicate).

3- Fruit firmness: Firmness of peal and pulp was measured with pressure test and expressed as $\mathrm{kg} / \mathrm{cm}^{2}$.

4- Peel percentage was calculated as a percentage of fruit weight, fruit juice was extracted and weighed then calculated as percentage of fruit weight for each treatment.

\section{Chemical characteristics of the fruit juice:}

1- TSS expressed as percentage.

2- Total sugars and reducing sugars percentage were determined according to a method of Land and Eynone outlined in the AOAC (1990).

3- Total acidity (\%) was determined as citric acid content as described in AOAC (1990).

4- TSS/total acidity ratio was calculated.
5- Ascorbic acid (Vitamin C) content as $\mathrm{mg} / 100 \mathrm{ml}$ juice was determined as described in AOAC (1990).

\section{Statistical Analysis:}

Randomized complete design with three replicates and with factorial was followed throughout the whole work and the least significant difference test (L.S.D) at 5\% level was used to differentiate means according to Snedecor and Cochran (1980).

\section{Results}

\section{1- The percentage of fruit weight loss and decay fruits:}

Data presented in Table (1) showed the effect of camphor and linin oils and imazalil (IMZ) on the percentage of weight loss and decay of Balady mandarin fruits during the cold storage in 2017 and 2018 seasons. It was obvious that results took similar trend during the two studied seasons.

Data in previously table clear that fruit weight loss, as well as, decayed fruits were markedly increased with advanced of cold storage period. These traits were slightly increased gradually from the beginning of cold storage till the $4^{\text {th }}$ month. After one or two months of cold storage in all oils or imazalil treatments the fruits were without any decay, respectively during the two studied seasons.

Weight loss and decay percentage increased during storage, reaching values of $(14.65 \&$ $15.35 \%)$ and $(48.33 \& 50.00 \%)$ in control fruits after four months. The weight loss was significantly lower at $6.12,8.65,11.8,8.17$ $\& 7.91 \%$ and $7.19,7.33,10.47,7.8,8.04 \%$ due to immerse the fruits with camphor oil, 
linin oil, imazalil, camphor plus imazalil, linin plus imazalil during the two studied seasons, respectively. Also, the corresponding values of decay percentage was attained $(16.67,15.03,8.33,6.67 \&$ $8.33)$ and . $(15.00,16.67,8.33,9.00 \&$ $5.00 \%$ ), respectively.

In response of oils and imazalil treatments, it was apparent that all treatments significantly reduced the fruit weight loss percentage and undesirable fruit percentage during cold storage for four months compared with control. Using oil singly or plus imazalil had the best results, which gave the least percentage of fruit weight loss. On other hand, using imazalil singly or plus oil gave the least percentage of decayed fruits compared to control and other treatments. No significant differences in weight loss due to use oil singly or plus IMZ. Also, no significant differences in decayed fruit percentage due to use IMZ or plus oil. The decrement percentage of weight loss percentage attained(68.09,63.81,47.67,67.44 and $66.02 \%$ av. of the two studied seasons) due to camphor oil $\left(\mathrm{T}_{1}\right)$, linin oil $\left(\mathrm{T}_{2}\right), \mathrm{IMZ}$ $\left(\mathrm{T}_{3}\right)$, camphor oil plus IMZ $\left(\mathrm{T}_{4}\right)$, linin oil plus IMZ $\left(\mathrm{T}_{5}\right)$ compared to untreat one $\left(\mathrm{T}_{\mathbf{6}}\right)$, respectively. The corresponding decrement percentage decayed fruits values attained (70.60, 68.03, 90.72, $91.53 \& 93.24 \%$ av. of the two studied seasons), respectively.

The variation in reduction of fruit loss and decayed fruits depend on the material coating. Use any oil gave the least percentage of fruit weight loss, whereas, IMZ induce a least percentage of decayed fruits compared to the other treatments and untreat one (control).
The results indicated that immersing with oil plus IMZ proved effective in reducing the percentage of weight loss and decayed fruits as well as keeping the Balady mandarin fruits for longest period.

\section{2-The percentage of peel and juice contents:}

The date introduced in Table (2) disclosed that the peel percentage increased and gradually from beginning of cold storage till the $4^{\text {th }}$ month. The lowest fruit peel percentage recorded at the beginning of cold storage, but the highest ones resulted after four month of cold storage. Contrary, fruit juice percentage significantly decreased by extending cooling storage duration. The highest values recorded at beginning of cold storage, whereas, the least ones was recorded at the end of cold storage course.

All oils treatments tended to induce reduce the fruit peel percentage, as well as, increased the fruit juice percentage compared to control. The least significant fruit peel percentage $(25.17 \& 25.83 \%)$ and the highest significant fruit juice percentage (34.15 \& 33.23\%) were found due to use camphor oil during the two studied seasons, respectively. On other hand, the highest fruit peel percentage $(27.37 \& 27.94 \%)$ and lowest fruit juice percentage $(32.43 \&$ $31.57 \%$ ) were recorded on untreated fruits (control) during the two studied seasons, respectively. No significant differences due to camphor oil and other oil singly or plus IMZ treatments. The decrement percentage of fruit peel percentage attained 8.04,7.82 $5.30,7.78 \& 7.78 \%$ and $7.55,7.09,4.90$, $7.73 \& 7.77 \%$ due to $\mathrm{T}_{1}$ to $\mathrm{T}_{5}$ compared to $\mathrm{T}_{6}$ during the two studied seasons, respectively. On other hand, the increment 
$\%$ of fruit juice percentage attained ( 5.30 , compared to $\mathrm{T}_{6}$ during the two studied $5.08,3.58,4.90 \& 5.08 \%)$ and $(5.25$, seasons, respectively.

$5.54,4.15,5.38 \& 5.19 \%)$ due to $\mathrm{T}_{1}$ to $\mathrm{T}_{5}$

Table 1. Effect of post-harvest treatments on fruit weight loss (\%) and decay (\%) of Balady mandarin fruits during cold storage in 2017 and 2018 seasons.

Fruit weight loss \% Decay \%

$\begin{array}{llllllllllll}\mathrm{P}_{0} & \mathrm{P}_{1} & \mathrm{P}_{2} & \mathrm{P}_{3} & \mathrm{P}_{4} & \mathrm{M} & \mathrm{P}_{0} & \mathrm{P}_{1} & \mathrm{P}_{2} & \mathrm{P}_{3} & \mathrm{P}_{4} & \mathrm{M}\end{array}$

\begin{tabular}{lllllllllllll}
\hline \multicolumn{2}{l}{ Season 2017 } & & & & & & & & & & & \\
Ca & 0 & 0.42 & 1.57 & 4.01 & 6.12 & 2.42 & 0 & 0 & 6.67 & 8.33 & 16.67 & 6.33 \\
L & 0 & 0.73 & 2.1 & 3.4 & 8.65 & 2.98 & 0 & 0 & 5.0 & 6.67 & 15.03 & 6.01 \\
IM & 0 & 0.94 & 2.09 & 5.44 & 11.8 & 4.05 & 0 & 0 & 0 & 0 & 8.33 & 1.67 \\
Ca.IM & 0 & 0.69 & 0.74 & 3.17 & 8.17 & 2.55 & 0 & 0 & 0 & 0 & 6.67 & 1.33 \\
L.IM & 0 & 0.69 & 0.65 & 3.79 & 7.91 & 2.61 & 0 & 0 & 0 & 0 & 8.33 & 1.67 \\
C & 0 & 6.01 & 8.15 & 10.5 & 14.65 & 7.81 & 0 & 3.33 & 18.33 & 30 & 48.33 & 19.33 \\
M & 0 & 1.58 & 2.55 & 5.05 & 9.55 & & 0 & 0.56 & 5 & 7.5 & 17.23 & \\
LSD 5\% & P: & 1.23 & T: & 1.11 & TP: & 2.45 & P: & 1.11 & T: & 1.07 & TP: & 2.44 \\
\hline Season 2018 & & & & & & & & & & & & \\
Ca & 0 & 0.39 & 1.32 & 3.56 & 7.19 & 2.49 & 0 & 0 & 3.33 & 8.33 & 15 & 5.33 \\
L & 0 & 0.64 & 2.25 & 2.77 & 7.33 & 2.60 & 0 & 1.67 & 6.67 & 8.33 & 16.67 & 6.67 \\
IM & 0 & 1.34 & 2.23 & 6.04 & 10.47 & 4.02 & 0 & 0 & 0 & 1.67 & 8.33 & 2.0 \\
Ca.IM & 0 & 0.66 & 0.86 & 3.07 & 7.8 & 2.47 & 0 & 0 & 0 & 1.17 & 9 & 2.03 \\
L.IM & 0 & 0.74 & 0.82 & 3.56 & 8.04 & 2.63 & 0 & 0 & 0 & 0 & 5 & 1.0 \\
C & 0 & 5.56 & 7.36 & 9.76 & 15.35 & 7.61 & 0 & 6.67 & 16.67 & 28.33 & 50 & 20.33 \\
M & 0 & 1.56 & 2.47 & 0.84 & 9.36 & & 0 & 1.39 & 4.44 & 7.97 & 17.33 & \\
LSD 5\% & P: & 1.12 & T: & 0.99 & TP: & 2.25 & P: & 1.43 & T: & 1.28 & TP: & 2.88 \\
\hline
\end{tabular}

$\mathrm{Ca}=$ Camphor $\quad \mathrm{L}=$ Linen; $\quad \mathrm{IM}=$ Imazalil; $\quad$ Ca.IM= Camphor + Imazalil;

L.IM= Linin + Imazalil; $\quad \mathrm{C}=$ Control 
Table 2. Effect of post-harvest treatments on peel $\%$ and juice weight $\%$ of Balady mandarin fruits during cold storage in 2017 and 2018 seasons.

\begin{tabular}{|c|c|c|c|c|c|c|c|c|c|c|c|c|}
\hline & \multicolumn{6}{|c|}{ Peel \% } & \multicolumn{6}{|c|}{ Juice weight $\%$} \\
\hline & $\mathrm{P}_{0}$ & $\mathrm{P}_{1}$ & $\mathrm{P}_{2}$ & $\mathrm{P}_{3}$ & $\mathrm{P}_{4}$ & $\mathrm{M}$ & $\mathrm{P}_{0}$ & $\mathrm{P}_{1}$ & $\mathrm{P}_{2}$ & $\mathrm{P}_{3}$ & $\mathrm{P}_{4}$ & $\mathrm{M}$ \\
\hline \multicolumn{13}{|c|}{ Season 2017} \\
\hline $\mathrm{Ca}$ & 23.61 & 24.12 & 25.06 & 26.14 & 26.9 & 25.17 & 37.34 & 36.12 & 34.6 & 31.89 & 30.81 & 34.15 \\
\hline $\mathrm{L}$ & 23.61 & 24.49 & 24.95 & 25.9 & 27.18 & 25.23 & 37.34 & 36.28 & 34.86 & 31.58 & 30.34 & 34.08 \\
\hline $\mathrm{IM}$ & 23.61 & 25.08 & 26.31 & 26.97 & 27.65 & 25.92 & 37.34 & 35.61 & 33.84 & 31.05 & 30.12 & 33.59 \\
\hline Ca.IM & 23.61 & 24.38 & 25.11 & 26 & 27.11 & 25.24 & 37.34 & 36.3 & 34.29 & 31.69 & 30.50 & 34.02 \\
\hline L.IM & 23.61 & 24.56 & 24.85 & 26.1 & 27.08 & 25.24 & 37.34 & 36.18 & 34.40 & 31.90 & 30.56 & 34.08 \\
\hline $\mathrm{C}$ & 23.61 & 26.2 & 27.93 & 28.86 & 30.28 & 27.37 & 37.34 & 35.15 & 32.33 & 29.09 & 28.27 & 32.43 \\
\hline M & 23.61 & 24.81 & 25.70 & 26.66 & 27.70 & & 37.34 & 35.94 & 34.05 & 31.19 & 30.08 & \\
\hline LSD 5\% & P: & 1.78 & $\mathrm{~T}:$ & 1.61 & TP: & 3.64 & P: & 1.04 & $\mathrm{~T}:$ & 1.41 & TP: & 3.16 \\
\hline \multicolumn{13}{|c|}{ Season 2018} \\
\hline $\mathrm{Ca}$ & 22.72 & 24.81 & 26.1 & 27.43 & 28.11 & 25.83 & 36.58 & 35.1 & 33.64 & 31.05 & 29.8 & 33.23 \\
\hline $\mathrm{L}$ & 22.72 & 25.29 & 26.53 & 27.35 & 27.89 & 25.96 & 36.58 & 35.41 & 34.00 & 30.92 & 29.68 & 33.32 \\
\hline IM & 22.72 & 25.86 & 27.11 & 27.89 & 29.26 & 26.57 & 36.58 & 34.98 & 32.13 & 30.57 & 29.12 & 32.88 \\
\hline Ca.IM & 22.72 & 24.88 & 26.38 & 27.16 & 27.76 & 25.78 & 36.58 & 35.4 & 33 & 30.85 & 29.6 & 33.27 \\
\hline L.IM & 22.72 & 25.11 & 26 & 27.08 & 27.95 & 25.77 & 36.58 & 35.45 & 33 & 31.12 & 29.9 & 33.21 \\
\hline $\mathrm{C}$ & 22.72 & 27.68 & 28.76 & 29.44 & 31.1 & 27.94 & 36.58 & 34.25 & 31.18 & 28.39 & 27.43 & 31.57 \\
\hline M & 22.72 & 25.61 & 26.81 & 27.73 & 28.68 & & 36.58 & 35.10 & 33.33 & 29.73 & 29.24 & \\
\hline LSD $5 \%$ & P: & 1.62 & $\mathrm{~T}:$ & 1.44 & TP: & 3.22 & P: & 1.68 & $\mathrm{~T}:$ & 1.50 & TP: & 3.35 \\
\hline
\end{tabular}

$\mathrm{Ca}=$ Camphor; $\mathrm{L}=$ Linen; $\quad \mathrm{IM}=$ Imazalil; $\quad \mathrm{Ca} . \mathrm{IM}=$ Camphor + Imazalil; L.IM= Linin + Imazalil $; \quad \mathrm{C}=\mathrm{Control}$ 


\section{3- Fruit firmness:}

Data of peel and pulp firmness as affected by different post-harvest treatments are presented in Table (3). It is obvious from the data that results took similar trend during the two studied seasons. Fruit firmness is a major attribute that dictates the post-harvest life and quality of fruits.

Data disclosed that peel and pulp firmness significantly decreased by extending cooling storage duration. These traits were slightly decreased and gradually from the beginning of cold storage till the $4^{\text {th }}$ moth. The highest values were recorded at the beginning of storage, whereas, the least ones was recorded at the end of storage period. Using oil singly or plus IMZ lead to significantly increased peel and pulp firmness compared to control or IMZ alone. No significant differences were found due to use camphor oil or linin oil during both studied seasons.

Fruits coating with camphor oil had higher peel firmness $\left(1.08 \& 1.00 \mathrm{~kg} / \mathrm{cm}^{2}\right)$ and pulp firmness $\left(1.32 \& 1.32 \mathrm{~kg} / \mathrm{cm}^{2}\right)$ than control fruits, peel (1.01 \& 0.93), pulp (1.23 \& 1.23 $\left.\mathrm{kg} / \mathrm{cm}^{2}\right)$. The increment percentage of peel firmness (6.93 \& $7.53 \%)$ and pulp firmness $(7.32 \& 7.32 \%)$ due to use camphor oil compared to control during the two studied seasons, respectively.

\section{4- Fruit chemical properties:}

The data concerning the effect of various cold storage treatments on chemical constituents of Balady mandarin juice during 2017 and 2018 seasons are presented in Tables (4, 5 and 6). It was obvious from the data that results took similar trend during the three studied seasons.
In general view, data indicated that chemical juice quality in terms, total soluble solids, sugars contents and TSS/acid ratio significantly increased during storage duration up to $4^{\text {th }}$ month. On other hand, prolonging cooling storage for four month induce a gradually decrease of titratable acidity and ascorbic acid (V.C).

According of treatment effects, it is clear from the previously data that all treatments lead significant effects on chemical juice properties compared to control. Moreover, using oil singly or plus IMZ significantly decreased total soluble solids, sugars contents and TSS/acid ratio and (V.C) content compared to other untreat ones (control). Also, such treatment significantly increased the titratable acidity compared to control.

Moreover, the highest values of total soluble solids, sugar contents and TSS/acid and least values of total acidity and V.C content were recorded on untreated fruits at end of storage period compared with other treatments. On other hand, the least values of total soluble solids, sugar contents and TSS/acid and highest values of total acidity and V.C contents were recorded on fruits coating with oil singly or plus IMZ during the two studied seasons.

According to previous results, it could be concluded that using $10 \%$ camphor oil or $10 \%$ linin oil seemed to be the proper and ideal treatment to prolong cold storage of mandarin fruits without great reduction in fruit quality. 
Table 3. Effect of post-harvest treatments on peel firmness and pulp firmness $\left(\mathrm{kg} / \mathrm{cm}^{2}\right)$ of Balady mandarin fruits during cold storage in 2017 and 2018 seasons.

\begin{tabular}{|c|c|c|c|c|c|c|c|c|c|c|c|c|}
\hline & \multicolumn{6}{|c|}{ Pulp firmness $\mathrm{kg} / \mathrm{cm}^{2}$} & \multicolumn{6}{|c|}{ Peel firmness $\mathrm{kg} / \mathrm{cm}^{2}$} \\
\hline & $\mathrm{P}_{0}$ & $\mathrm{P}_{1}$ & $\mathrm{P}_{2}$ & $\mathrm{P}_{3}$ & $\mathrm{P}_{4}$ & M & $\mathrm{P}_{0}$ & $\mathrm{P}_{1}$ & $\mathrm{P}_{2}$ & $\mathrm{P}_{3}$ & $\mathrm{P}_{4}$ & M \\
\hline \multicolumn{13}{|c|}{ Season 2017} \\
\hline $\mathrm{Ca}$ & 1.54 & 1.51 & 1.34 & 1.21 & 1.03 & 1.32 & 1.28 & 1.23 & 1.1 & 0.98 & 0.84 & 1.08 \\
\hline $\mathrm{L}$ & 1.54 & 1.52 & 1.32 & 1.22 & 1.02 & 1.32 & 1.28 & 1.24 & 1.09 & 0.99 & 0.83 & 1.08 \\
\hline IM & 1.54 & 1.46 & 1.22 & 1.10 & 0.93 & 1.25 & 1.28 & 1.20 & 1.02 & 0.91 & 0.77 & 1.03 \\
\hline Ca.IM & 1.54 & 1.50 & 1.34 & 1.22 & 1.03 & 1.32 & 1.28 & 1.22 & 1.1 & 0.97 & 0.83 & 1.08 \\
\hline L.IM & 1.54 & 1.52 & 1.34 & 1.20 & 1.10 & 1.33 & 1.28 & 1.24 & 1.11 & 0.99 & 0.82 & 1.09 \\
\hline $\mathrm{C}$ & 1.54 & 1.44 & 1.19 & 1.07 & 0.90 & 1.23 & 1.28 & 1.17 & 1.0 & 0.89 & 0.75 & 1.01 \\
\hline M & 1.54 & 1.49 & 1.29 & 1.17 & 1.00 & & 1.28 & 1.22 & 1.07 & 0.95 & 0.81 & \\
\hline LSD $5 \%$ & P: & 0.06 & $\mathrm{~T}:$ & 0.06 & TP: & 0.13 & P: & 0.06 & $\mathrm{~T}:$ & 0.05 & TP: & 0.11 \\
\hline \multicolumn{13}{|c|}{ Season 2018} \\
\hline $\mathrm{Ca}$ & 1.50 & 1.48 & 1.37 & 1.18 & 1.05 & 1.32 & 1.15 & 1.13 & 1.03 & 0.89 & 0.78 & 1.0 \\
\hline $\mathrm{L}$ & 1.50 & 1.47 & 1.36 & 1.17 & 1.04 & 1.31 & 1.15 & 1.12 & 1.04 & 0.89 & 0.79 & 1.0 \\
\hline IM & 1.50 & 1.40 & 1.27 & 1.09 & 0.97 & 1.22 & 1.15 & 1.06 & 0.95 & 0.81 & 0.70 & 0.93 \\
\hline Ca.IM & 1.50 & 1.46 & 1.38 & 1.18 & 1.04 & 1.31 & 1.15 & 1.11 & 1.04 & 0.88 & 0.8 & 1.0 \\
\hline L.IM & 1.50 & 1.48 & 1.36 & 1.15 & 1.06 & 1.31 & 1.15 & 1.13 & 1.03 & 0.9 & 0.89 & 1.02 \\
\hline $\mathrm{C}$ & 1.50 & 1.41 & 1.25 & 1.07 & 0.95 & 1.23 & 1.15 & 1.05 & 0.94 & 0.80 & 0.71 & 0.93 \\
\hline M & 1.50 & 1.45 & 1.33 & 1.14 & 1.02 & & 1.15 & 1.10 & 1.00 & 0.876 & 0.78 & \\
\hline LSD $5 \%$ & P: & 0.07 & $\mathrm{~T}:$ & 0.06 & TP: & 0.13 & P: & 0.07 & $\mathrm{~T}:$ & 0.06 & TP: & 0.13 \\
\hline
\end{tabular}

$\mathrm{Ca}=$ Camphor; $\mathrm{L}=$ Linen; $\quad \mathrm{IM}=$ Imazalil; $\quad \mathrm{Ca} . \mathrm{IM}=$ Camphor + Imazalil;

L.IM= Linin + Imazalil; $\quad \mathrm{C}=$ Control 
Table 4. Effect of post-harvest treatments on total soluble solids (TSS) \% and titratable acidity $\%$ of Balady mandarin fruits juice during cold storage in 2017 and 2018 seasons.

\begin{tabular}{|c|c|c|c|c|c|c|c|c|c|c|c|c|}
\hline & \multicolumn{6}{|c|}{ TSS \% } & \multicolumn{6}{|c|}{ Acidity \% } \\
\hline & $\mathrm{P}_{0}$ & $\mathrm{P}_{1}$ & $\mathrm{P}_{2}$ & $\mathrm{P}_{3}$ & $\mathrm{P}_{4}$ & $\mathrm{M}$ & $\mathrm{P}_{0}$ & $\mathrm{P}_{1}$ & $\mathrm{P}_{2}$ & $\mathrm{P}_{3}$ & $\mathrm{P}_{4}$ & $\mathrm{M}$ \\
\hline \multicolumn{13}{|c|}{ Season 2017} \\
\hline $\mathrm{Ca}$ & 10.34 & 10.48 & 10.73 & 11.18 & 11.70 & 10.89 & 1.42 & 1.35 & 1.24 & 1.16 & 1.07 & 1.25 \\
\hline $\mathrm{L}$ & 10.34 & 10.56 & 10.86 & 11.26 & 11.65 & 10.93 & 1.42 & 1.36 & 1.24 & 1.18 & 1.1 & 1.26 \\
\hline IM & 10.34 & 10.58 & 11.43 & 11.8 & 12.15 & 11.26 & 1.42 & 1.32 & 1.2 & 1.07 & 0.96 & 1.19 \\
\hline Ca.IM & 10.34 & 10.5 & 10.91 & 11.03 & 11.28 & 10.81 & 1.42 & 1.38 & 1.22 & 1.18 & 1.06 & 1.25 \\
\hline L.IM & 10.34 & 10.4 & 10.75 & 11.1 & 11.4 & 10.80 & 1.42 & 1.35 & 1.25 & 1.18 & 1.08 & 1.26 \\
\hline $\mathrm{C}$ & 10.34 & 10.85 & 11.68 & 12.10 & 12.71 & 11.54 & 1.42 & 1.3 & 1.16 & 1.03 & 0.9 & 1.16 \\
\hline M & 10.34 & 10.56 & 11.06 & 11.40 & 11.81 & & 1.42 & 1.34 & 1.22 & 1.13 & 1.03 & \\
\hline LSD 5\% & P: & 0.52 & $\mathrm{~T}$ : & 0.45 & TP: & 1.01 & P: & 0.09 & $\mathrm{~T}:$ & 0.08 & TP: & 0.17 \\
\hline \multicolumn{13}{|c|}{ Season 2018} \\
\hline $\mathrm{Ca}$ & 10.86 & 10.73 & 11.05 & 11.83 & 11.90 & 11.24 & 1.48 & 1.36 & 1.28 & 1.2 & 1.14 & 1.29 \\
\hline $\mathrm{L}$ & 10.68 & 10.76 & 11.10 & 11.99 & 12.05 & 11.32 & 1.48 & 1.36 & 1.3 & 1.22 & 1.15 & 1.30 \\
\hline IM & 10.68 & 10.82 & 11.38 & 12.1 & 12.41 & 11.49 & 1.48 & 1.36 & 1.21 & 1.15 & 1.03 & 1.25 \\
\hline Ca.IM & 10.68 & 10.81 & 11.1 & 11.66 & 11.99 & 11.25 & 1.48 & 1.37 & 1.29 & 1.22 & 1.06 & 1.28 \\
\hline L.IM & 10.68 & 10.79 & 11.18 & 11.72 & 12.02 & 11.28 & 1.48 & 1.35 & 1.28 & 1.23 & 1.15 & 1.30 \\
\hline $\mathrm{C}$ & 10.68 & 11.16 & 11.83 & 12.38 & 12.88 & 11.83 & 1.48 & 1.32 & 1.16 & 1.01 & 0.98 & 1.19 \\
\hline M & 10.68 & 10.84 & 11.27 & 11.95 & 12.21 & & 1.48 & 1.35 & 1.25 & 1.17 & 1.09 & \\
\hline LSD $5 \%$ & P: & 0.44 & $\mathrm{~T}:$ & 0.40 & TP: & 0.89 & P: & 0.10 & $\mathrm{~T}:$ & 0.19 & TP: & 0.44 \\
\hline
\end{tabular}

$\mathrm{Ca}=$ Camphor; $\mathrm{L}=$ Linen; $\quad \mathrm{IM}=$ Imazalil; $\quad \mathrm{Ca} . \mathrm{IM}=$ Camphor + Imazalil;

L.IM= Linin + Imazalil; $\quad \mathrm{C}=$ Control 
Table 5. Effect of post-harvest treatments on TSS/acid ratio and vitamin C content of Balady mandarin fruits juice during cold storage in 2017 and 2018 seasons.

\begin{tabular}{|c|c|c|c|c|c|c|c|c|c|c|c|c|}
\hline & \multicolumn{6}{|c|}{ TSS/acid ratio } & \multicolumn{6}{|c|}{ V.C content $\mathrm{mg} /$ ascorbic acid/100 ml juice } \\
\hline & $\mathrm{P}_{0}$ & $\mathrm{P}_{1}$ & $\mathrm{P}_{2}$ & $\mathrm{P}_{3}$ & $\mathrm{P}_{4}$ & M & $\mathrm{P}_{0}$ & $\mathrm{P}_{1}$ & $\mathrm{P}_{2}$ & $\mathrm{P}_{3}$ & $\mathrm{P}_{4}$ & M \\
\hline \multicolumn{13}{|c|}{ Season 2017} \\
\hline $\mathrm{Ca}$ & 7.28 & 7.76 & 8.65 & 9.63 & 10.93 & 8.85 & 41.24 & 40.1 & 38.85 & 38.23 & 37.29 & 39.14 \\
\hline $\mathrm{L}$ & 7.28 & 7.76 & 8.75 & 9.54 & 10.59 & 8.79 & 41.24 & 40.0 & 38.8 & 38.11 & 37.46 & 39.12 \\
\hline $\mathrm{IM}$ & 7.28 & 8.01 & 9.52 & 11.02 & 12.65 & 9.70 & 41.24 & 39.51 & 37.48 & 36.36 & 34.11 & 37.74 \\
\hline Ca.IM & 7.28 & 7.60 & 8.94 & 9.34 & 10.64 & 8.76 & 41.24 & 39.85 & 38.9 & 38.2 & 37.30 & 39.01 \\
\hline L.IM & 7.28 & 7.70 & 8.60 & 9.40 & 10.55 & 8.71 & 41.24 & 39.93 & 38.8 & 38.44 & 37.25 & 39.13 \\
\hline $\mathrm{C}$ & 7.28 & 8.19 & 9.89 & 11.57 & 13.90 & 10.17 & 41.24 & 37.00 & 35.22 & 34.23 & 31.69 & 35.98 \\
\hline M & 7.28 & 7.84 & 9.06 & 10.09 & 11.54 & & 41.24 & 39.40 & 38.04 & 37.20 & 35.86 & \\
\hline LSD $5 \%$ & P: & 0.96 & $\mathrm{~T}:$ & 0.83 & TP: & 1.92 & P: & 1.61 & $\mathrm{~T}:$ & 1.40 & TP: & 3.26 \\
\hline \multicolumn{13}{|c|}{ Season 2018} \\
\hline $\mathrm{Ca}$ & 7.21 & 7.91 & 8.67 & 9.99 & 10.78 & 8.92 & 44.10 & 43.25 & 41.7 & 39.35 & 38.64 & 41.41 \\
\hline $\mathrm{L}$ & 7.21 & 7.88 & 8.50 & 9.69 & 10.28 & 8.72 & 44.10 & 43.32 & 41.63 & 39.29 & 38.71 & 41.41 \\
\hline IM & 7.21 & 7.95 & 9.40 & 10.52 & 12.33 & 9.49 & 44.10 & 42.48 & 40.25 & 38.18 & 37.11 & 40.42 \\
\hline Ca.IM & 7.21 & 7.89 & 8.60 & 9.55 & 11.31 & 8.92 & 44.10 & 43.18 & 41.7 & 39.42 & 38.5 & 41.38 \\
\hline L.IM & 7.21 & 7.99 & 8.73 & 9.52 & 10.45 & 8.78 & 44.10 & 43.2 & 41.68 & 39.51 & 38.89 & 41.48 \\
\hline $\mathrm{C}$ & 7.21 & 8.30 & 10.02 & 12.05 & 12.42 & 10.01 & 44.10 & 40.55 & 37.14 & 35.12 & 33.90 & 38.12 \\
\hline M & 7.21 & 7.99 & 8.99 & 10.23 & 11.17 & & 44.10 & 42.63 & 40.69 & 38.48 & 37.62 & \\
\hline LSD $5 \%$ & P: & 0.99 & $\mathrm{~T}:$ & 0.86 & TP: & 1.96 & P: & 1.83 & $\mathrm{~T}:$ & 1.61 & TP: & 3.68 \\
\hline
\end{tabular}

$\mathrm{Ca}=$ Camphor; $\quad \mathrm{L}=$ Linen; $\quad \mathrm{IM}=$ Imazalil; $\quad$ Ca.IM= Camphor + Imazalil;

L.IM= Linin + Imazalil; $\quad \mathrm{C}=$ Control 
Table 6. Effect of post-harvest treatments on total and reducing sugars of Balady mandarin fruits juice during cold storage in 2017 and 2018 seasons.

\begin{tabular}{|c|c|c|c|c|c|c|c|c|c|c|c|c|}
\hline & \multicolumn{6}{|c|}{ Total sugars } & \multicolumn{6}{|c|}{ Reducing sugars } \\
\hline & $\mathrm{P}_{0}$ & $\mathrm{P}_{1}$ & $\mathrm{P}_{2}$ & $\mathrm{P}_{3}$ & $\mathrm{P}_{4}$ & $\mathrm{M}$ & $\mathrm{P}_{0}$ & $\mathrm{P}_{1}$ & $\mathrm{P}_{2}$ & $\mathrm{P}_{3}$ & $\mathrm{P}_{4}$ & $M$ \\
\hline \multicolumn{13}{|c|}{ Season 2017} \\
\hline $\mathrm{Ca}$ & 7.45 & 7.60 & 7.67 & 7.95 & 8.3 & 7.79 & 3.08 & 3.08 & 3.14 & 3.26 & 3.32 & 3.18 \\
\hline $\mathrm{L}$ & 7.45 & 7.61 & 7.75 & 7.98 & 8.27 & 7.81 & 3.08 & 3.1 & 3.17 & 3.28 & 3.34 & 3.19 \\
\hline IM & 7.45 & 7.72 & 8.11 & 8.4 & 8.67 & 8.07 & 3.08 & 3.16 & 3.33 & 3.46 & 3.58 & 3.32 \\
\hline Ca.IM & 7.45 & 7.58 & 7.75 & 7.83 & 8.20 & 7.76 & 3.08 & 3.1 & 3.15 & 3.22 & 3.3 & 3.17 \\
\hline L.IM & 7.45 & 7.55 & 7.66 & 7.88 & 8.08 & 7.72 & 3.08 & 3.09 & 3.15 & 3.24 & 3.35 & 3.18 \\
\hline $\mathrm{C}$ & 7.45 & 7.80 & 8.18 & 8.52 & 8.88 & 8.17 & 3.08 & 3.15 & 3.36 & 3.5 & 3.62 & 3.34 \\
\hline M & 7.45 & 7.64 & 7.85 & 8.09 & 8.40 & & 3.08 & 3.11 & 3.22 & 3.33 & 3.42 & \\
\hline $\begin{array}{c}\text { LSD } \\
5 \%\end{array}$ & P: & 0.38 & $\mathrm{~T}$ : & 0.33 & TP: & 0.75 & P: & 0.11 & $\mathrm{~T}:$ & 0.10 & TP: & 0.23 \\
\hline \multicolumn{13}{|c|}{ Season 2018} \\
\hline $\mathrm{Ca}$ & 7.63 & 7.73 & 7.78 & 8.49 & 8.76 & 8.08 & 3.26 & 3.31 & 3.38 & 3.6 & 3.68 & 3.45 \\
\hline $\mathrm{L}$ & 7.63 & 7.71 & 7.93 & 8.43 & 8.67 & 8.07 & 3.26 & 3.3 & 3.39 & 3.59 & 3.67 & 3.44 \\
\hline IM & 7.63 & 7.8 & 8.16 & 8.63 & 9.08 & 8.26 & 3.26 & 3.36 & 3.52 & 3.71 & 3.89 & 3.55 \\
\hline Ca.IM & 7.63 & 7.76 & 7.85 & 8.4 & 8.62 & 8.05 & 3.26 & 3.32 & 3.4 & 3.58 & 3.68 & 3.45 \\
\hline L.IM & 7.63 & 7.73 & 7.91 & 8.38 & 8.65 & 8.06 & 3.26 & 3.31 & 3.42 & 3.58 & 3.88 & 3.49 \\
\hline $\mathrm{C}$ & 7.63 & 7.92 & 8.67 & 8.97 & 9.53 & 8.52 & 3.26 & 3.39 & 3.6 & 3.78 & 3.95 & 3.60 \\
\hline M & 7.63 & 7.78 & 8.05 & 8.54 & 8.85 & & 3.26 & 3.33 & 3.45 & 3.64 & 3.79 & \\
\hline $\begin{array}{c}\text { LSD } \\
5 \%\end{array}$ & P: & 0.46 & $\mathrm{~T}:$ & 0.41 & TP: & 0.93 & P: & 0.10 & $\mathrm{~T}:$ & 0.09 & TP: & 0.20 \\
\hline
\end{tabular}

$\mathrm{Ca}=$ Camphor $; \mathrm{L}=$ Linen; $\quad \mathrm{IM}=$ Imazalil; $\quad \mathrm{Ca} \cdot \mathrm{IM}=$ Camphor + Imazalil;

L.IM= Linin + Imazalil; $\quad \mathrm{C}=$ Control 


\section{Discussion}

Handling and storage is an important to fruit production and its quality. Fresh weight loss and fruit decay percentage were increased by extending storage duration. The loss of water from fruits during storage is a substantial problem due to shrinkage and weight loss, thus, the fruits could be damaged and loss its quality (BenYehoshua, 2005). The fruit weight decrease due to its respiratory process, the transference of humidity and some processes of evaporation of moisture inside the fruits (Hassan et al., 2014).

Coating were used widely in fruits to reduce dehydration and water loss, prevents shriveling in fruit skin. Coating can act as a semi-permeable barrier against oxygen, carbon dioxide, moisture, so, they can reduce the rate of the respiration, water loss and oxidation reaction (Park, 1999). Oil coating have been reported to close stomata which resulted in suppressed respiration rate and minimized weight loss of fruit under cold storage (Regnier et al., 2014 and Gouda-Fatma El-Zhhraa and BadawySabah, 2016).

Decay of mandarin fruits during storage are mostly the cause of Penicillium sp. Moreover, the function of coating is a partial retention of gas exchange through the fruit peel and inhibition of the action of ethylene. Inhibition can give more prevention against post-harvest decay and water loss from the peel so decreasing the incidence of decay during storage (Abd El-Motty and ElFaham, 2013; Mansour, 2015 and GoudaFatma El-Zhhraa and Badawy-Sabah, 2016).

The decreasing in decay percentage of treated fruits was probably due to the effects of coating on delaying senescence, which makes the commodity more vulnerable to pathogenic infection as a result of loss of cellular or tissue integrity (Patricia et al., 2005 and Barakat et al., 2015).

Higher firmness in treated fruits by oils compared to untreat ones (control) might be attributed to the reduced hydrolysis of soluble starch (Zaghloul et al., 2011). Also may be due to a reduction membrane lipid peroxidation by enhancing the capacity of cells to scavenge reactive oxygen species (Lin et al., 2008).

The results are in accordance with those obtained by Baldwin et al. (1995), Rodov et al. (2001), Plooy et al. (2009), Abd ElMotty and El-Faham (2013), Mansour (2015), Barakat et al. (2015) and GoudaFatma El-Zhhraa and Badawy-Sabah (2016).

It is known that during storage there is an antagonistic relation between TSS and acidity, while water loss and TSS were increased and the acidity was decreased due to the process of respiration in fruits, because fruits consume acidity for respiration thereby total soluble solids increase more than acidity, thus TSS/acid ratio increases. The decrease in acid content is caused by the use of acids in the fruit as a source of energy and the conversion of organic acids to form sugar (Wills et al., 1998).

Lower level of ascorbic acid in untreated fruits might be due to increased respiration. Ascorbic acid is susceptible to oxidative deterioration or the formation of dehydroascorbic acid (Wills et al., 1998). Coating reduces oxygen levels and results in lower phenolic and antioxidant compounds during storage (Tietel et al., 2010). 
The above mentioned findings are in accordance with those obtained by Saucedo et al. (2007), Plooy et al. (2009), Tietel et al. (2010), Abd El-Motty and El-Faham (2013) and Mansour (2015).

\section{Conclusion}

Generally, from the above mentioned results, it could be concluded that the most effective treatments in maintaining quality properties of Balady mandarin fruits after four months were $10 \%$ camphor oils plus IMZ.

\section{Conflict of interest}

The authors hereby declare that no competing and conflict of interests exist.

\section{References}

A.O.A.C. (1990) 'Methods of Analysis. Association of Official Analytical Chemists' $14^{\text {th }}$ Ed., Washington, USA.

Abd El-Motty, Z. and El-Faham, S.Y. (2013) 'Effect of Oil Coating and Different Wrapping Materials on Prolonging Storage Periods of Florida Prince Peach Fruits'. Journal of Applied Sciences Research, 9(4), pp. 29272937.

Ahmed, D.M., El-Shami, S.M. and ElMallah, M.H. (2007) 'Jojoba oil as a novel coating for exported Valencia orange fruit. Part 1. The use of trans (isomerized) jojoba oil'. Am. Eurasian. J. Agric. Environ. Sci., 2, pp. 173-181.

Badawy-Ibtesam, F.M., Nashwa Sallam, M.A., Ibrahim, A.R. and Asran, M.R. (2011) 'Efficacy of some essential oils on controlling green mold of orange and their effects on post-harvest quality parameters'. Plant Physiology J., 10 (4), pp.

168-174.
Bajpai, V.K., Shukla, S. and Kang, Sc. (2008) 'Chemical composition and antifungal activity of essential oil and various extract of Silene armeria L'. Bioresource Technol. 99 (18), pp. 8903-8908. doi.:

10.1016/j.biortech.2008.04.060

Bakhtiarizade, M. and Souril, M.K. (2019). Beneficial effects of rosemary, thyme and tarragon essential oils on postharvest decay of Valencia oranges. Chem. Biol. Technol. Agric., 6(9), pp. 1-8. doi: 10.1186/s40538-019-0146-3

Baldwin, E.A., Nisperos-Carriedo, M., Shaw, P.E. and Burns, J.K. (1995) 'Effect of coatings and prolonged storage conditions on fresh orange flavor volatiles, degrees, brix and ascorbic acid levels'. Journal of Agricultural and Food Chemistry, 43 (5), pp. 1321-1331. doi: 10.1021/jf00053a037

Barakat, M.R., Abeer T. Mohsen and Azhar A.A. Mohamed (2015) 'Effect of some natural oils and salicylic acid on fruit quality of Valencia orange during storage'. Journal of Horticultural Sci. and Ornamental Plants, 7 (2), pp. 6670.

Ben-Yehoshua, S. (2005) 'Individual, seal packaging of fruits and vegetables in plastic film-a new post-harvest technique'. HortScience 20, pp. 32-37.

Bull, C.T., Stack, J.P. and Smilanick, J.L. (1997) 'Pseudomonas syringae strains ESC-10 and ESC-11 survive in wounds citrus and control green and blue mold of citrus'. Biol. Control, 8, pp. 81-88. doi:10.1006/bcon.1996.0476

Eman, A.A.A. and Magda, A.A. (2006) 'Effect of some oil emulsions and wax treatment on prolonging storage period of Washington navel orange fruits and its volatile components'. Journal of Applied Sciences Research, 2(7), pp. 405-417. 
Ennab, H.A., Mervat A. El-Shemy and Shamel M. Alam-Eldein (2020) 'Salicylic, acid and putrescine to reduce post-harvest storage problems and maintain quality of Murcott mandarin fruit. Agronomy 2020, 10, 115; doi:10.3390/agronomy10010115.

Gouda-Fatma El-Zhhraa, M. and BadawySabah, M. (2016) 'Effect of coating and wrapping materials on storage behavior of Balady mandarin fruits'. Assiut J. Agric. Sci., 47 (5), pp. 138-150. doi: 10.21608/ajas.2016.2065

Hassan, Z.H., Lesmayati, S., Qomariah, R. and Hasbianto, A. (2014) 'Effect of wax coating applications and storage temperatures on the quality of tangerine citrus (Citrus reticulata) var. Siam Banjar'. Int. Food Res. J., 21(2), pp. 641-648. doi: 10.17660/actahortic.2013.1011.51

Janisiewicz, W.J. and Korsten, L. (2002) 'Biological control of post-harvest diseases of fruits'. Ann. Rev. Phytopathology, 40, pp. 411-444. doi: 10.1146/annurev.phyto.40.120401.1301 58

Kader, A.A. (2002) 'Modified atmospheres during transport and storage. In postHarvest Technology of Horticultural Crops (Ed)'. Univ. Calif. Div. Agr. Nat. Res. Publ. 3311 Oakland, CA pp. 135144.

Klieber, A., Scott, S.E. and Wuryatmo, E. (2002) 'Effect of method of application on antifungal efficacy of citrus against post-harvest spoilage fungi of citrus in culture'. Aust. Plant Pathol., 31, pp. 329-332. doi: 10.1071/ap02034

Lin, L., Wang, B.G., Wang, M., Coa, J., Zhang, J., Wu, Y. and Jilang, W. (2008) 'Effect of achitosan-based coating with ascorbic acid on post harvest quality and core browning of "Yali" pears (Pyrus bretschneideri Redh)'. J. Sci.
Food Agric., 88 (5), pp. 877-884. doi.:10.1002/jsfa.3164

Mahmoud, T.S.M., Yassin, N.M.A. and Shaaban, F.K.M. (2017). 'Influence of Postharvest Application with Chitosan and some Natural Plant Extracts on Storage Life and Quality Attributes of Navel Orange Fruits during Cold Storage.', Midd. East J. of Agric. Res, 6(2), pp.330-339.

Mansour, A.H. (2015) 'Effect of pre and post-harvest treatments on fruit characteristics of Balady mandarin (Citrus reticulata L.) during storage'. Ph.D. Thesis, Fac. of Agric., Assiut Univ., Egypt.

Mansour, A.H., El-Salhy, A.M., Mostafa, F.M. and Samia G. El-Orabi (2014) 'Effect of intermittent warming treatments on the storability of Balady mandarin (Citrus reticulate L.) fruits'. Assiut J. Agric. Sci., 45 (4), pp. 45-58. doi: 10.21608/ajas.2014.3499

Mehrdad, J. and Sajad, F. (2012) 'Post harvest treatments on shelf life of sweet orange "Valencia"'. Journal of Medicinal Plants Research, 6(11), pp. 2117-2124. doi:10.5897/jmpr11.1065

Park, H.J. (1999) 'Development of advanced edible coatings for fruits'. Trends Food Sci. Technol., 10, pp. 254-260. doi:10.1016/s0924-2244(00)00003-0

Patricia, S., Palmu, T. and Grosso, C.R.F. (2005) 'Effect of edible wheat glutenbased films and coating on refrigerated strawberry (Fragaria ananassa) quality'. Postharvest Biol. Technol., 36, pp. 199-208. doi:10.1016/j.postharvbio.2004.12.003

Plooy, W., Regnier, T. and Combrinck, S. (2009) 'Essential oil amended coatings as alternatives to synthetic fungicides in citrus post-harvest management'. Postharvest Biology and Technology, 53, 
pp.

117-122.

doi:10.1016/j.postharvbio.2009.04.005

Poppe, L., Vanhoutte, S. and Hofte, M. (2003) 'Modes of action of Pantoea agglomerans CPA-2, an antagonist of post-harvest pathogens on fruits'. Eur. $J$. Plant Pathol., 109, pp. 963-973. doi:10.1023/b:ejpp.0000003747.41051. 9f

Ragsdale, N.N. and Sisler, H.D. (1994) 'Social and political implications of managing plant diseases with decreased availability of fungicides in the United States'. Ann. Rev. Phytopathol., 32, pp. 545-557.

doi: 10.1146/annurev.py.32.090194.002553

Regnier, T., Combrink, S., Veldman, W. and Du Plooy, W. (2014) 'Application of essential oils as multi-target fungicides for the control of Geotrichum citriaurantii and other post harvest pathogens of citrus'. Ind. Crop Prod., 61, pp. 151-159. doi.:10.1016/j.indcrop.2014.05.052

Rodov, V., Agar, T., Peretz, J., Nafussi, B., Kim Jong, and Ben-Yehoshua, S. (2001) 'Effect of combined application of heat treatments and plastic packaging on keeping quality of "oroblanco" fruit (Citrus grandis L. Xc. Paradisimacr)'. Post-harvest Biology and Technology, 20 (3), pp. 287-294. (c.f. Hort. Abst. 71 (4), p. 2911). doi.:10.1016/s09255214(00)00129-0

Saucedo, P.S., Jasso-Cantu, D., VenturaSabrevilla, J., Saenz-Galindo, A., Rodriguez-Herrera, R. and Aguilar, C.N. (2007) 'Effect of canenlla wax with natural antioxidants on the shelf life quality of fresh cut fruits'. J. of Food Quality, 30 (5), pp. 823-836. doi:10.1111/j.1745-4557.2007.00165.x

Snedecor, G.W. and Cochran, W.G. (1980) 'Statistical Methods. $7^{\text {th }}$ ed.', Iowa State Univ. Press, Amer. Iowa, U.S.A., pp. 50 .
Taslima, Ayesha A.N., Rahman, M.A., Arfin, M.S, Islam, M.N. and Azmat Ullah, M. (2020). Effect of novel coconut oil and beeswax edible coating on postharvest quality of lemon at ambient storage. Journal of Agriculture and Food Research, 2 (2020), 100019. doi:10.1016/j.jafr.2019.100019

Tietel, Z., Lewinsohn, E., Feldmesser, E., Fallik, F. and Porat, R. (2010) 'Effect of wax coatings and post-harvest storage on sensory quality and aroma volatile composition of "Mor" mandarins'. J. of Sci. of Food Agric., 90 (6), pp. 9951007. doi:10.1002/jsfa.3909

Tripathi, P. and Duby, N.K. (2004) 'Exploitation of natural products as an alternative strategy to control post harvest fungal ratting of fruit and vegetable'. Post-harvest Biol. Technol., 32, pp. 335-345. doi: 10.1016/j.postharvbio.2003.11.005

Wills, R.B.H., Me Glasson, W.B., Graham, D. and Joyce, D. (1998) 'Post-harvest Introduction to the physiology and handling of fruits, vegetable and ornamentals'. $4^{\text {th }}$ New South Wales University Press Limited, Kensington, England.

Zaghloul, A.E., Abd El-Naiem, A.E. and Hamdy, E.E. (2011) 'Application of activated jasmine oil on navel orange trees b-improving storability and shelflife'. J. Agric. Res. Kafer El-Sheikh Univ., 37(2), pp. 370-384. 